\title{
Review of "One Database at a Time: PubMed for Expert Searchers and Systematic Reviews"
}

\author{
Alexandria Q. Wilson, MSIS, AHIP \\ Assistant Professor, Research and Learning Services Librarian \\ Preston Medical Library \\ The University of Tennessee Graduate School of Medicine \\ Knoxville, Tennessee \\ http://orcid.org/0000-0002-2649-8252
}

As the interest in systematic review expertise grows, the Medical Library Association Technology in Education and Systematic Review Caucuses came together to cosponsor a webinar series called "One Database at a Time" that examines and discusses how to use a different database for systematic searches. The Technology in Education Caucus (TEC) acknowledged the expertise available in the Systematic Review Caucus and approached them about co-sponsoring the series. The caucuses hope to provide both expert searching and systematic review tips for health science librarians and informationists.

The first session, focused on the PubMed database, was offered on March 23. Moderated by Margaret Foster and led by panelists Alyssa Grimshaw, Liz Suelzer, and LaTeesa James, the session covered a variety of topics, from building your systematic search in PubMed to systematic review software.

The session began with setting up a systematic search in PubMed. Panelists recommended starting with MeSH (Medical Subject Headings). Then, they recommended combining $\mathrm{MeSH}$ terms with keywords to facilitate translating the strategy to databases that do not use MeSH vocabulary and to catch any results that are not indexed with their MeSH terms yet.

Another key piece of advice was to utilize "search details." This allows searchers to see how PubMed translated the search. This even includes warnings if a search term does not map correctly or is pulling in irrelevant terms (e.g., the word "refusal" mapping to refuse or garbage).

While this session mainly focused on PubMed, there were topics covered that anyone completing a systematic review could benefit from, even if they do not use PubMed for their systematic search. The next section covered the documentation process of the search and guidelines to follow, such as PRISMA and the Equator Network. Once the search has been documented appropriately, panelists stressed the importance of using a citation manager, regardless of searching PubMed or not, to export and manage 
those results. The pros and cons of citation managers EndNote (Clarivate Analytics), Mendeley (Elsevier), and Zotero were discussed.

Systematic review software options of Covidence, Rayyan, and DistillerSR were briefly introduced before a formal Q\&A session, although the moderator and panelists engaged in questions from the attendees throughout the webinar. The chat feature of the webinar and the Q\&A session were moderated by Kay Hogan Smith and Tara Brigham. Questions and answers brought up important discussions comparing PubMed to OVID/Medline, signing up for search alerts versus re-running the entire search to update a systematic review, reproducibility concerns, and the "ideal" number of databases to search, if such a number exists.

Future sessions in this series will be determined via popular vote, and OVID/Medline is planned for the second session. This webinar recording was edited by Julia Stumpff, who also led the advertising efforts. To watch the recording in full or access the transcript, please visit https://iu.mediaspace.kaltura.com/media/t/1 shw3x5ld. 